\title{
Build a two-way road for health industry: the current circum- stance of translational medicine in China
}

\author{
LENG FangWei \\ Institute of Biophysics, Chinese Academy of Sciences, Beijing 100101, China
}

Received April 16, 2012; accepted July 20, 2012; published online September 22, 2012

Citation: $\quad$ Leng F W. Build a two-way road for health industry: the current circumstance of translational medicine in China. Sci China Life Sci, 2012, 55: 931-932, doi: 10.1007/s11427-012-4377-9

Translational medicine is a new emerging medical practice comprising multidisciplinary research from basic sciences to clinical medicine. It is regarded as a two-way road: bench to bedside and bedside to bench, playing important roles in communications between basic and clinical scientists [1]. As a developing country with more than 1.3 billion people, China bears a complex disease spectrum. It is thus urgent for China to develop translational medicine to facilitate prediction, prevention, diagnosis, and treatment of diseases. With the energetic supports of governments and scientists, translational medicine in China has developed rapidly in recent years. The 12th issue of Science China Life Sciences in 2011 organizes a special issue-Translational medicine in China I: Perspectives from Chinese physicians and scientists [2], focusing on translational medicine in China with five reviews, making a comprehensive introduction to the current circumstance of translational medicine in China.

With increasing attention and rapid development of biological research, many remarkable basic science discoveries about life have been achieved. Unfortunately, due to the limited communications between basic and clinical scientists, these valuable laboratory discoveries are difficult to be translated into clinical applications. This has opened up the demand for bridging between basic science and clinical use. The concept of translational medicine has emerged in recent years under this circumstance, aiming to facilitate the conversion of laboratory discoveries into clinical applications. The first issue of the Journal of Translational Medicine in

email: fangweihaha7@gmail.com
2003 formally declared translational medicine as a new discipline. Since then, translational medicine has developed rapidly and widely. Scientists all around the world have made many successful studies in translational medicine [3-6], greatly enhancing our understanding of translational medicine, and paving the way to more fruitful translational results. Actually, even before the appearance of the concept of translational medicine, there have already been many successful translational studies based on the bedsideto-bench-to-bedside translational strategy. The review entitled "Translational research: Lessons from past research, growing up nowadays, and development goal in future" by Zhang et al. [7] provides many successful examples of these classical translational studies, including the Framingham Heart Study and the development of statins to percutaneous coronary intervention treatment. Another review entitled "Liu Shih-Hao: Pioneer of translational medicine in China" by Li [8] reviews the research, teaching and clinical work of Liu Shih-Hao, who is regarded as the pioneer of translational medicine in China. Dr. Liu's early work in calcium and phosphorus metabolism is a well-known success story of translational medicine, which is still important and revelatory until now.

As a country with the largest population in the world, China bears a complex disease spectrum, from communicable diseases such as hepatitis B virus, tuberculosis and acquired immunodeficiency syndrome (AIDS) to chronic diseases including cancer, heart disease and hypertension. With the ultimate goal to develop more effective preventive/therapeutic approaches and improve clinical outcomes 
and health levels, translational medicine is therefore essential for China's health system. The first review entitled "Translational medicine should translate medical science and technology into health care for everyone in China" by Chen and Zhou [9] indicates that translational medicine should focus on patients and the general population, points out the importance of translational research for China's health industry, and also discusses proper principles for development of translational medicine in China. With support of the governments and scientists, especially financial supports, translational medicine in China has developed rapidly in recent years. The first Symposium on Translational Medicine in 2007 and the Sino-America Symposium on Clinical and Translational Research in June 2010 emphasize the commitment of China to the rapid development of translational medicine [10]. As the wave of translational medicine sweeping across the world, increasingly more excellent Chinese physicians and scientists have emerged, who have conducted remarkable work, enhancing the development of translational medicine. To take one example, the review entitled "Overview the theory on molecular targeted therapy, its features and future direction, and contributions in the field from Chinese scientists" by Liu et al. [11] displays the contributions of Chinese scientists to molecular targeted therapy (MTT), which has promising potential to kill tumors and induce tumor cells to differentiate into normal cells, leading to cure of the patients.

It is noteworthy that translational medicine in China has its own characteristics: moving traditional Chinese medicine (TCM) from empirical to evidence-based practicing. As widely practiced healing theories and practices for almost 2000 years, TCM is still popular in China now. There are many successful cases of translation from traditional Chinese medicine, providing Chinese clinicians with an important interpretation of translational medicine. The review entitled "Translational medicine: What is in a name from the perspective of Chinese clinicians?" by Zeng and Zhong [12] describes these successful examples of translation from traditional Chinese medicine, including the use of artemisinin in the management of malaria, the use of arsenic in the management of leukemia, and artificial infection with malaria parasites for lung cancer therapy [13-15].

Although translational medicine has developed rapidly in recent years, there remain several obstacles, including lack of sufficient funding, high cost and slow results, shortage of researchers, inadequate samples, and incompatible data- bases $[16,17]$. Further development of translational medicine requires more sources of funding and education, and the most important strategy to promote translational research is to decrease the cost by increasing its efficiency. For China, development of translational medicine will largely depend on collaboration between China and other countries, sharing the understanding, methodologies, research protocols and resources, and development. It is believed that translational medicine in China will realize a leapfrog development in the near future.

1 Marincola F M. Translational medicine: a two-way road. J Transl Med, 2003, 24: 1

2 Jiang C Y. Translational medicine in China I: Perspectives from Chinese physicians and scientists. Sci China Life Sci, 2011, 54: 1071-1073

3 Planas A M, Traystman R J. Advances in translational medicine 2010. Stroke, 2011, 42: 283-284

4 Moskowitz M A, Lo E H, Iadecola C. The science of stroke: mechanisms in search of treatments. Neuron, 2010, 67: 181-198

5 Schwartz M, Shechter R. Systemic inflammatory cells fight off neurode-generative disease. Nat Rev Neurol, 2010, 6: 405-410

6 Abe T, Shimamura M, Jackman K, et al. Key role of CD36 in Toll-like receptor 2 signaling in cerebralischemia. Stroke, 2010, 41: 898-904

7 Zhang Z Q, Chen H Z, Liu D P. Translational research: Lessons from past research, growing up nowadays, and development goal in future. Sci China Life Sci, 2011, 54: 1085-1088

8 Li N S. Liu Shih-Hao: Pioneer of translational medicine in China. Sci China Life Sci, 2011, 54: 1089-1095

9 Chen Z, Zhou G B. Translational medicine should translate medical science and technology into health care for everyone in China. Sci China Life Sci, 2011, 54: 1074-1076

10 Wang X, Wang E, Marincola F M. Translational medicine is developing in China: a new venue for collaboration. J Transl Med, 2011, 9: 3

11 Liu Q, Zhang X S, Zeng Y X. Targeted and personalized therapy for cancer: Theory and practice in China. Sci China Life Sci, 2011, 54: 1081-1084

12 Zeng G Q, Zhong N S. Translational medicine: What is in a name from the perspective of Chinese clinicians? Sci China Life Sci, 2011, 54: 1077-1080

13 Artemisinin antimalaria coordinating research group. Artemisinin: a novel sesquiterpene lactone (in Chinese). Chin Sci Bull, 1977, 3: 142

14 Zhang T D, Zhang P F, Wang S R, et al. A preliminary clinical observation with use of ailing injection in 6 cases of leukemia (in Chinese). Heilongjiang Med, 1973, 3: 66-67

15 Chen L, He Z, Qin L, et al. Antitumor effect of malaria parasite infection in a murine Lewis lung cancer model through induction of innate and adaptive immunity. PLoS ONE, 2011, 6: e24407

16 Mankoff S P, Brander C, Ferrone S, et al. Lost in translation: obstacles to translational medicine. J Transl Med, 2004, 2: 14

17 Littman B H, Di Mario L, Plebani M, et al. What's next in translational medicine? Clin Sci (Lond), 2007, 112: 217-227

Open Access This article is distributed under the terms of the Creative Commons Attribution License which permits any use, distribution, and reproduction in any medium, provided the original author(s) and source are credited. 